\title{
Fazit zum sexuellen Missbrauch durch katholische Geistliche

\author{
Zusammenfassung der Erkenntnisse aus den \\ KFN-Befragungen
}

Dirk Baier, Sandra Fernau, Deborah F. Hellmann, Christian Pfeiffer

\section{Einleitung}

Das Projekt, über dessen Befunde in den Beiträgen dieses Sammelbandes berichtet wurde, hat es dank der Unterstützung der Betroffenen des Missbrauchs durch katholische Geistliche in bislang einzigartiger Weise möglich gemacht, die Belastungen und Bewältigungsstrategien der Betroffenen, das Vorgehen der Täterinnen bzw. Täter und sonstige Umstände des Missbrauchs wissenschaftlich zu analysieren. An der mittels eines Fragebogens durchgeführten Studie beteiligten sich 113 Personen, an der die persönliche Biografie sowie die subjektive Verarbeitung in den Fokus nehmenden Interviewstudie 15 Personen. Ist für Deutschland bislang die empirische Beschäftigung mit dem Forschungsthema grundsätzlich eher die Ausnahme, so gilt dies in besonderer Weise für die Kombination der Forschungsstrategien der quantitativen und der qualitativen Befragung. Die Beiträge des Sammelbands verdeutlichen, dass die Verbindung unterschiedlicher methodischer Herangehensweisen erkenntnisförderlich ist. Nachfolgend sollen zentrale Befunde der vorgestellten Analysen zusammengefasst und diskutiert werden (II. bis XI.). Die dabei gewählte Reihenfolge beinhaltet keine Aussage über den Stellenwert eines Befundes. Es handelt sich allein um eine Auflistung, die auch anders gereiht hätte vorgenommen werden können.

Die Anzahl an Befragten, die den entsprechenden Auswertungen zugrunde lag (104 bzw. 15 Personen), ist eher gering. Den Autorinnen und Autoren dieses Sammelbandes erschien es dennoch wichtig, zu dieser für sozialwissenschaftliche Studien vergleichsweise kleinen Stichprobe Auswertungen vorzunehmen. Zu begründen ist dies damit, dass jeder ausgefüllte und zurückgesandte Fragebogen die Biografie eines Menschen erzählt, dem sehr belastende Erlebnisse widerfahren sind. Die Aussagen jedes einzelnen Befragten sind für sich allein genommen bereits von hohem 
wissenschaftlichem Interesse. Eine Bündelung der Aussagen, die dadurch ermöglicht wurde, dass mehr als 100 Betroffene dem Aufruf zur Teilnahme an der Studie folgten, ist besonders erkenntnisförderlich. $\mathrm{Zu}$ danken ist an dieser Stelle daher noch einmal allen Betroffenen, die die Untersuchung durch ihre Bereitschaft, sehr persönliche Erlebnisse mitzuteilen, unterstützt haben.

\section{Die sexuellen Missbrauchserfahrungen sind für einen Großteil der Betroffenen auch noch nach Jahrzehnten mit starken psy- chischen Beeinträchtigungen verbunden.}

Für den Durchschnitt aller Betroffenen gilt, dass das Erleben des sexuellen Missbrauchs fast fünf Jahrzehnte zurückliegt. Diese Erfahrung bezieht sich dabei auf die späte Kindheit: Die erste Missbrauchshandlung wurde im Mittel im elften Lebensjahr gemacht, die letzte im 14. Lebensjahr. Zeigt sich, dass bereits im direkten Anschluss an die Missbrauchserfahrung vielfach emotionale, körperliche und verhaltensrelevante Beeinträchtigungen festzustellen sind (bspw. gab mehr als ein Viertel der befragten Betroffenen an, dass sie unmittelbar danach selbstverletzendes Verhalten ausgeführt hätten), so werden die langfristigen psychischen Konsequenzen der Missbrauchserfahrungen gerade durch die Betrachtung des aktuellen Zustands deutlich: Für fast zwei Drittel (64.4\%) aller Betroffenen ergeben sich Hinweise auf das Vorliegen einer Posttraumatischen Belastungsstörung. Das heißt, es wird sich immer wieder an das belastende Ereignis erinnert. Bestimmte Aktivitäten, Orte usw. werden gemieden, um belastenden Erinnerungen aus dem Weg zu gehen. Die betroffenen Personen sind übermäßig reizbar und neigen zu Wutausbrüchen.

Darüber hinaus zeigen die Auswertungen, dass $83.3 \%$ der Betroffenen als stark psychisch belastet eingestuft werden müssen. Jeweils fast drei Viertel der betroffenen Personen sind ängstlich oder depressiv, weisen verstärkt paranoides Denken oder Psychotizismus auf. Starke psychische Belastungen sind wiederholt bei Betroffenen von sexueller Gewalt festgestellt worden (z. B. Whitelock, Lamb \& Rentfrow, 2013). Die hier erzielten hohen Beeinträchtigungswerte auch Jahrzehnte nach den Vorfällen geben aber einen Hinweis darauf, dass der sexuelle Missbrauch durch katholische Geistliche eine besondere Deliktskategorie darstellt. Dies hängt zunächst mit dem spezifischen Täterkreis zusammen: Die Handlungen wurden von Personen in einer hohen sozialen Stellung ausgeführt, die aufgrund ihrer ethisch-moralischen und religiösen Autorität eine besondere 
Vertrauensposition innehatten sowie bei den Eltern hohes Ansehen genossen (Benkert \& Doyle, 2009; Plante 1996).

Hinzu kommt, dass viele Betroffene multiplen Missbrauch erlebt haben. Multipel bedeutet einerseits, dass Übergriffe von mehreren Geistlichen ausgeführt worden sind; immerhin fast ein Drittel der Betroffenen bestätigt dies. Multipel bedeutet andererseits, dass die Mehrheit der betroffenen Personen unterschiedlichen Missbrauchshandlungen in mehrheitlich wiederholter Ausübung ausgesetzt war. Im Durchschnitt wurden über fünf Missbrauchshandlungen berichtet (vom Stellen intimer Fragen bis hin zu verschiedenen Formen sexueller Praktiken). Für alle diese einzelnen Handlungen gilt, dass der kleinere Teil der Betroffenen ein einmaliges Erlebnis angibt, die größere Anzahl also mehrfach eine Handlung erlebt hat. So berichten zum Beispiel $35.8 \%$ der Befragten von einer Penetration durch die Täterin bzw. den Täter; bei $9.5 \%$ war dies einmal der Fall, bei $26.3 \%$ mehrmals.

\section{Die Bewältigung der psychischen Beeinträchtigungen gelingt in unterschiedlicher Weise, generell aber eher schlecht.}

Die Betroffenen setzen sich aktiv mit den psychischen Beeinträchtigungen auseinander. Dies spiegelt sich unter anderem darin wider, dass sich $61.1 \%$ von ihnen an eine Therapeutin bzw. einen Therapeuten gewandt haben, $37.3 \%$ an eine Beratungsstelle und $13.7 \%$ an eine Selbsthilfegruppe. Dies ist mehrheitlich mit größerem zeitlichen Abstand zu den Missbrauchserlebnissen geschehen. Der Erfolg der Aufarbeitung des Erlebten ist zugleich sehr unterschiedlich. Generell gilt, dass Personen, die verschiedene und häufig stattfindende Missbrauchshandlungen erlebt haben, aktuell ein schlechteres Befinden zeigen als Personen, die wenigen Übergriffen ausgesetzt waren. Zudem spielt das Geschlecht eine Rolle: Weibliche Befragte weisen tendenziell häufiger eine Posttraumatische Belastungsstörung auf als männliche Befragte. Wichtig ist daneben das Ausmaß der individuellen Resilienz: Betroffene, die trotz der Erlebnisse eine hohe Widerstandskraft bewahren konnten, erweisen sich als geringer psychisch belastet. Dies ist nicht überraschend, verweist aber auf die weiterführende Frage, welchen Personen es grundsätzlich gelingt, unter widrigen Bedingungen Optimismus, Selbstwirksamkeit und Ähnliches zu bewahren.

Sehr instruktiv für die Frage der Bewältigung von sexuellen Missbrauchserfahrungen sind die Ergebnisse der Betroffeneninterviews. Diese 
illustrieren, wie schwer es den Betroffenen fällt, biografische Umgangsformen mit dem Erlebten auszubilden, die dazu beitragen, mit den Erfahrungen zurechtzukommen und sie auf eine Weise einzuordnen, dass sie keine zu starke Belastung darstellen. Besonders auffällig ist in diesem $\mathrm{Zu}$ sammenhang die auch Jahrzehnte nach den Taten bei vielen Interviewten bestehende Angst vor einer Stigmatisierung durch das familiäre und soziale Umfeld sowie der oftmals hiermit verbundene antizipierte Verlust der Zugehörigkeit zur kirchlichen Gemeinde bei einer Offenbarung des Missbrauchs. Eine offene Thematisierung und Aufarbeitung der Viktimisierungserfahrungen innerhalb der Glaubensgemeinschaft und teils auch im familiären Kreis bleibt hier aus.

Dies weist darauf hin, dass gerade die durch die religiöse Sozialisation mitbedingte starke emotionale Verbundenheit und Loyalität zur Kirchengemeinde einigen Betroffenen eine Verarbeitung des sexuellen Missbrauchs im Zuge einer Aufdeckung und aktiven Hilfesuche im sozialen und kirchlichen Umfeld erschwert. Hierbei handelt es sich um eine spezifische Aufdeckungsproblematik innerkirchlichen sexuellen Missbrauchs. Darüber hinaus zeigen sich in den Schilderungen der Interviewten verschiedene Formen der Umdeutung der Missbrauchsvorkommnisse: Zum Teil klagen sich die Betroffenen an, selbst Schuld an den Übergriffen zu tragen. Oder sie bagatellisieren die teilweise schweren sexuellen Missbrauchshandlungen, denen sie ausgesetzt waren bzw. ordnen die Vorfälle dahingehend ein, dass sie diese selbst mit initiiert hätten. Es kommt auch vor, dass sie das Geschehen religiös begreifen und beispielsweise als Teil eines ,göttlichen Plans“ umdeuten. Somit findet eine positive, sinnstiftende Umbewertung des sexuellen Missbrauchs statt.

Zwar stand die Frage, inwieweit diese Umgangsformen funktional im Sinne einer erfolgreichen Bewältigung sind oder nicht, bei den Interviewauswertungen nicht im Zentrum der Betrachtung. Die berichteten langfristigen und teils gravierenden Folgen der Missbrauchserfahrung(en) lassen jedoch auf eine zumindest als ambivalent einzustufende Wirkung der rekonstruierten Umgangsformen auf die Verarbeitung der erlebten sexuellen Übergriffe schließen. Die hohen Belastungswerte, die für die Gruppe der Betroffenen in der Fragebogenerhebung ermittelt werden konnten, deuten ebenfalls darauf hin, dass es sich tendenziell um wenig funktionale Bewältigungsstrategien handelt. 


\section{Der sexuelle Missbrauch fand häufig im Kontext von Heimen oder Internaten statt. Der Missbrauch hier weist zugleich eine eigene Qualität auf.}

Als Orte der Übergriffe sind häufig institutionelle Kontexte benannt worden: Von allen Betroffenen geben $29.8 \%$ an, dass der Missbrauch in einem Heim erfolgt wäre; in $18.3 \%$ der Fälle handelte es sich um einen Missbrauch im Internat. Damit ist jede bzw. jeder zweite Betroffene in institutionellen Kontexten missbraucht worden. Die andere Hälfte der Betroffenen lebte zum Zeitpunkt des Übergriffs bei den (Groß-)Eltern (inkl. Adoptiv-/Pflegeltern). Für die männlichen Betroffenen hat der Internatskontext dabei eine höhere Relevanz als für die weiblichen: $22.1 \%$ der männlichen Betroffenen erlebten hier den Missbrauch, aber lediglich $3.8 \%$ der weiblichen Betroffenen. Dies hängt damit zusammen, dass katholische Internate insbesondere in den 1950er bis 1970er Jahren als Eliteschulen (teilweise auch für potenzielle Priesteramtskandidaten) vor allem Jungen vorbehalten waren.

Der insgesamt hohe Anteil an Missbrauchsbetroffenen in institutionellen Zusammenhängen dürfte auch dadurch erklärbar sein, dass - wie zum Beispiel Keupp, Straus, Mosser, Gmür und Hackenschmiedt (2013) herausgestellt haben - es sich bei diesen Kontexten um von der Außenwelt isolierte Eigenwelten handelte, in denen eigenständige, streng hierarchisch aufgebaute Regel- und Handlungssysteme galten, die Betreuenden und Lehrenden eine hohe Machtposition innehatten, Sexualität tabuisiert wurde usw. Hinzu kommt, dass es sich gerade bei Heimkindern um Personen handelt, die in der Vergangenheit wenig Zuwendung erlebt haben und dadurch eine besondere Anfälligkeit für die Annäherungsversuche von Erwachsenen aufweisen (z. B. MacLean, 2003).

Übereinstimmend mit diesen Überlegungen deuten die vorliegenden Befunde darauf hin, dass der sexuelle Missbrauch in Institutionen als besonders schwerwiegend einzustufen ist. Dies gilt vor allem für Betroffene, die innerhalb eines Heimkontexts viktimisiert worden sind: Nicht nur, dass Betroffene in Heimen bereits bei der Initiierung des Missbrauchs besonders häufig bedroht worden sind und physische Gewalt erlebt haben; ihnen sind auch häufiger die körperlich schwersten Missbrauchsformen und in diesem Zusammenhang weitere schwere Formen physischer Gewalt widerfahren. So berichten die Betroffenen aus dem Heimkontext (58.1\%) fast doppelt so häufig von schwerer physischer Gewalt in $\mathrm{Zu}-$ sammenhang mit dem sexuellen Missbrauch wie Betroffene aus dem In- 
ternatskontext (30.0\%) und mehr als sechsmal häufiger als Betroffene aus dem nicht-institutionellen Kontext (9.4\%).

Auffällig häufig sind Betroffene in Heimen zudem von unterschiedlichen Täterinnen bzw. Tätern in unabhängigen Zeiträumen viktimisiert worden. Sexueller Missbrauch durch mehrere Täterinnen bzw. Täter in einem Zeitraum kam sowohl in Internaten als auch in Heimen in etwa jedem vierten bzw. fünften Fall vor und damit in etwas dreimal häufiger als im nicht-institutionellen Kontext.

In Einklang mit diesen Ergebnissen ging das Erleben sexuellen Missbrauchs im Heimkontext im Vergleich zum Internatskontext und zum nicht-institutionellen Kontext unmittelbar mit stärkeren körperlichen Folgen, aber auch langfristig mit stärkerer psychischer Belastung einher.

\section{Sexueller Missbrauch durch katholische Geistliche ist in Bezug auf den institutionellen Kontext nachweislich ein rückläufiges Phänomen.}

Die US-amerikanische Forschung hat ergeben, dass der Höhepunkt der Missbrauchszahlen in den 1970er Jahren zu beobachten war; danach setzte ein starker Rückgang ein (Terry et al., 2011). Dies wird insbesondere mit Veränderungen in der klerikalen Ausbildung, einer höheren Sensibilität für den sexuellen Missbrauch sowie der gesellschaftlichen Entwicklung hin zu einer liberaleren Sexualmoral erklärt. Die ermittelten Zahlen aus Deutschland bestätigen einen Rückgang des Missbrauchs: Von allen Betroffenen berichten $26.0 \%$, dass der Missbrauch in den Jahren 1949 bis 1959 stattfand; in den Zeitraum 1980 bis 1993 fielen $16.4 \%$ der hier erfassten Missbräuche (1960 bis 1969: 29.8 \%, 1970 bis 1979: 27.9 \%). Eine differenzierte Analyse belegt jedoch, dass der Rückgang hauptsächlich den institutionellen Kontext betrifft: In den Zeitraum 1980 bis 1993 fallen nur noch $7.8 \%$ der in Heimen bzw. Internaten erfolgten Missbrauchsvorfälle, in den Zeitraum 1949 bis 1959 hingegen noch $35.3 \%$. Bei den nichtinstitutionellen Missbrauchsvorfällen ist zwar ebenfalls ein leichter Rückgang festzustellen; dieser fällt aber deutlich geringer aus. Mit den vorliegenden Daten kann zugleich nichts über die Entwicklung nach 1993 ausgesagt werden, da sich keine betroffene Person gemeldet hat, deren Missbrauch in diesen Zeitraum fiel. Dies bedeutet nicht, dass es solche Betroffene nicht gibt. Aus den Angaben zum Offenbarungsverhalten geht hervor, dass gewöhnlich eine lange Zeit zwischen dem Missbrauch und der Offenbarung gegenüber anderen Personen vergeht. Verlässliche Zah- 
len zur gegenwärtigen Verbreitung sexuellen Missbrauchs durch katholische Geistliche wird es insofern erst in Zukunft geben können. Dass es möglicherweise zu einem weiteren Rückgang des sexuellen Missbrauchs durch katholische Geistliche gekommen ist, belegt ein Befund einer 2011 durchgeführten, deutschlandweit repräsentativen Befragung von 16- bis 40-jährigen Personen: Dort hatte nur eine einzige Person angegeben, durch einen katholischen Geistlichen missbraucht worden zu sein (Stadler, Bieneck \& Pfeiffer, 2012, S. 40).

Der Umstand, dass der Missbrauch im institutionellen Kontext zurückgeht, lässt Zweifel an der Erklärung zu, dass primär innerkirchliche Veränderungen hierfür verantwortlich sind. Eine klerikale Ausbildung durchlaufen in institutionellen Kontexten tätige Personen ebenso wie in nichtinstitutionellen Kontexten tätige Personen. Wahrscheinlicher als Erklärung ist deshalb, dass Heime in ihrem Stellenwert hinsichtlich der Erziehung von Waisen oder aus anderen Gründen hier untergebrachten Kindern und Jugendlichen quantitativ an Bedeutung verloren haben. Der Rückgang des Missbrauchs kann somit möglicherweise in erster Linie durch die vermehrte Schließung von Heimen in den vergangenen Jahrzehnten erklärt werden. Die Thematisierung der Problematik innerhalb der katholischen Kirche im Zuge des Bekanntwerdens der Missbrauchsfälle im Jahr 2010 (u. a. durch die Einsetzung von Missbrauchsbeauftragten) lässt überdies nicht ohne Weiteres die Folgerung zu, dass die Sensibilität seit den 1980er Jahren zugenommen hätte. Dies ist wohl erst seit den letzten Jahren der Fall.

\section{Bei den Täterinnen bzw. Tätern handelt es sich vorwiegend um einzelne männliche Personen, die meist die Stellung eines Pries- ters innehatten.}

In $67.6 \%$ der berichteten Fälle war die Täterin bzw. der Täter ein einzelner Geistlicher der katholischen Kirche. Bei insgesamt $66.3 \%$ der Täter handelte es sich um einen Priester, Pfarrer oder Kaplan; die zweithäufigste Nennung bezieht sich auf Angehörige eines Ordens. In den Fällen, in denen das Geschlecht der Täterin bzw. des Täters explizit aufgrund der Betroffenenangaben zu ermitteln war, kann gesagt werden, dass es sich zu $89.0 \%$ ausschließlich um einen oder mehrere männliche/n Täter handelte; ein Missbrauch durch ausschließlich weibliche Täterinnen wurde nur in $3.7 \%$ der Fälle berichtet. 
Die angeführten Missbrauchshandlungen weisen eine große Bandbreite auf und reichen vom Stellen intimer Fragen zur Sexualität (71.7 \% der Betroffenen berichten hiervon) bis hin zur Aufforderung, sexuelle Handlungen mit anderen Kindern oder Jugendlichen zu vollziehen (14.3 \%). Häufig schreckten die Täterinnen bzw. Täter zudem nicht davor zurück, mit Drohungen und Gewalt zu arbeiten. Auch Alkohol- und Drogenkonsum spielte in Zusammenhang mit dem Missbrauch eine Rolle: In jedem fünften Fall wurde angegeben, dass die Täterin bzw. der Täter unter dem Einfluss von Alkohol und Drogen gestanden hat. Diese Befunde bestätigen weitestgehend das Täterbild, das sich aus Studien zum sexuellen Missbrauch beispielsweise aus den USA oder Österreich ergibt (John Jay College, 2004; Lueger-Schuster, 2012).

\section{Der sexuelle Missbrauch ist auch ein Ergebnis des Missbrauchs der Stellung der Täterinnen bzw. Täter, ihrer körperlichen Überlegenheit und der sozial-räumlichen Gegebenheiten.}

Die Auswertungen machen deutlich, dass es zum sexuellen Missbrauch gekommen ist, weil die Täterinnen bzw. Täter in verschiedener Hinsicht ihre privilegierte Lage ausgenutzt, diese missbraucht haben. Dies lässt sich zunächst anhand ihrer Stellung illustrieren: So waren sie mehrheitlich (68.3 \% der Fälle) der Familie bekannt und genossen hier ein hohes Ansehen. In über 80 \% der Fälle, in denen die Täterin bzw. der Täter der Familie bekannt war, wird berichtet, dass diese eine hohe Meinung von ihr bzw. ihm hatte bzw. glücklich und stolz in Bezug auf den Kontakt der Betroffenen zur Täterin bzw. zum Täter war. Die Täterin bzw. der Täter hatte also Zugang zur Familie, die ihm vertrauensvoll begegnete, und dies vor allem auch deshalb, weil sie bzw. er aufgrund der ethisch-moralisch herausgehobenen Position eine gesellschaftliche Autorität darstellte (Benkert \& Doyle, 2009; Plante 1996).

Jene besondere Stellung wurde zugleich hinsichtlich der mit ihr verbundenen religiösen Funktion missbraucht. Dies spiegelt sich unter anderem in der Tatsache wider, dass Missbrauchshandlungen zum Beispiel zu $21.2 \%$ in Zusammenhang mit der Beichte, zu $20.2 \%$ vor oder nach dem Gottesdienst und zu 10.6\% im Rahmen des Messdienerunterrichts stattfanden. Jede bzw. jeder dritte Befragte gab an, dass der Missbrauch explizit in religiöse Handlungen eingebettet worden wäre. Die Täterinnen oder Täter konnten also auf Begründungsweisen ihres Handelns zurückgreifen, die anderen Missbrauchstäterinnen und -tätern im nicht-kirchlichen Kon- 
text nicht zur Verfügung stehen. Dies gilt ebenso hinsichtlich der entworfenen Bedrohungsszenarien. Jede zweite betroffene Person gab beispielsweise an, dass ihr gedroht worden wäre, dass Gott sie bestrafe, wenn sie über den Missbrauch spräche. Gerade in hochreligiösen sozialen Gemeinschaften und bei Vorliegen einer starken individuellen Religiosität dürften solche Drohungen ihren Erfolg nicht verfehlen.

Es sind im Bereich des innerkirchlichen sexuellen Missbrauchs also spezifische Täterstrategien auszumachen, die sich als religiöse Manipulation der Betroffenen bezeichnen lassen. Derartige religiöse Neutralisierungstechniken werden verschiedentlich auch in den qualitativen Interviews geschildert. Bei einer Interviewten wurde der Missbrauch zum Beispiel unter Verweis auf die Notwendigkeit einer Teufelsaustreibung legitimiert. Zudem liefern die Interviews Belege, wie der Missbrauch in religiöse Praktiken, beispielsweise das Beichten oder das Buße-Tun eingebunden wurde. Diese Verbindung des Religiösen, Heiligen mit dem sexuellen Missbrauch kann eine Erklärung dafür darstellen, warum es manchen Betroffenen tendenziell schlechter gelingt, das Erlebte zu bewältigen. So finden sich teils bis heute Spuren der religiösen Neutralisierungstechniken in den Erzählungen der Betroffenen, am deutlichsten in der Übernahme der Missbrauchsdeutung der Täterin bzw. des Täters. Die Analyse zeigt dabei, dass eine Abkehr von den von der Täterin bzw. dem Täter herangezogenen Deutungsmustern insbesondere durch eine Bindung der Betroffenen an katholische Glaubensvorstellungen erschwert wird.

Ferner missbrauchten die Täterinnen bzw. Täter häufig ihre körperliche Überlegenheit, wobei es diesbezüglich keine Unterschiede zu anderen Missbrauchstäterinnen und -tätern geben dürfte. Erwähnenswert ist dennoch, dass jede zweite betroffene Person davon berichtet, Drohungen von Seiten der Täterinnen bzw. Täter ausgesetzt gewesen zu sein; mindestens jede vierte betroffene Person gibt an, dass sie neben den sexuellen Missbrauchshandlungen auch physischer Gewalt ausgesetzt gewesen sei. Gewalt wurde daneben in $28.8 \%$ der Fälle bei der Initiierung der ersten Missbrauchshandlung eingesetzt.

Hinsichtlich der letzten Form der privilegierten Lage ist unklar, ob und wie die Täterinnen und Täter diese zu ihrem Vorteil eingesetzt haben. Gemeint ist, dass sich der Missbrauch mehrheitlich in Städten und Dörfern ereignete, in denen die Einwohnerinnen und Einwohner überwiegend katholischen Glaubens waren: 69.3 \% der Betroffenen bestätigen dies. Ein solches konfessionelles Milieu, das sich grundsätzlich allerdings auch in Gegenden, in denen Katholikinnen und Katholiken in der Minderheit sind, ausbilden kann, dürfte sich zumindest darauf auswirken, ob sich die Be- 
troffenen dritten Personen gegenüber offenbaren und eine Autorität eines strafbaren Verhaltens beschuldigen oder nicht. Ganz in diesem Sinne veranschaulichen die qualitativen Interviews, dass das subjektive Erleben eines großen Teils der stark in das kirchliche Gemeindeleben eingebundenen Betroffenen bis heute von Ängsten hinsichtlich möglicher negativer Folgen der Aufdeckung ihrer Erlebnisse geprägt ist: Es herrscht eine tief verankerte Angst vor ablehnenden Reaktionen, Schuldzuweisungen und Stigmatisierungen gerade auch durch das kirchliche Umfeld, was auf die besondere soziale Lage verweist, die die Täterinnen und Täter innerhalb der Gemeinschaft innehatten. Darüber hinaus könnte sich ein solches, stark katholisch geprägtes Milieu darauf auswirken, dass sich Täterinnen und Täter dazu ermutigt fühlen, aufgrund ihrer selbstwahrgenommenen Unantastbarkeit Straftaten zu begehen.

\section{Viele Personen im direkten Umfeld der Betroffenen haben vom Missbrauch gewusst; die bewusste Offenbarung erfolgte aber meist erst lange nach dem Missbrauch.}

Fast vier von fünf Betroffenen (78.6\%) geben an, dass Personen in ihrem nahen sozialen Umfeld vom sexuellen Missbrauch Kenntnis gehabt hätten: Betroffene, die den Missbrauch im institutionellen Kontext erlebt haben, bestätigen dies tendenziell häufiger als andere Betroffene $(86.0 \% \mathrm{zu}$ $71.7 \%$ ). Nichtsdestotrotz wurde der Missbrauch selten beendet, was damit zu erklären ist, dass es sich um ähnlich „machtlose“ Mitwisserinnen und Mitwisser wie die Betroffenen bzw. um ,mächtige“ Vertraute der Täterinnen und Täter handelte. In $49.5 \%$ der Fälle wussten beispielsweise andere Betroffene von dem sexuellen Missbrauch, in $21.4 \%$ hatten Freundinnen bzw. Freunde, Bekannte oder Mitschülerinnen und Mitschüler der Betroffenen davon Kenntnis. Bei Betroffenen aus institutionellen Kontexten liegen diese Anteile deutlich höher als bei anderen Betroffenen (für Freundinnen bzw. Freunde usw.: $32.0 \%$ zu $11.3 \%$; für andere Betroffene: $62.0 \%$ zu $37.7 \%$ ). Demgegenüber steht, dass in knapp einem Viertel der Fälle den Vorgesetzten oder Kolleginnen bzw. Kollegen der Täterinnen und Täter die Übergriffe bekannt waren; bei etwas mehr als jedem fünften Fall wussten andere Erwachsene in der Institution, Personen innerhalb der Gemeinde oder Mittäterinnen bzw. Mittäter Bescheid. Die Täterinnen und Täter wurden damit häufig gedeckt; eine adäquate Verfolgung von bekannt gewordenen Vorfällen gab es nicht. Allerdings ist nicht allein ein Versagen der jeweiligen Institutionen im Besonderen oder der katholi- 
schen Kirche im Allgemeinen zu konstatieren, sondern auch ein Versagen von familiären Vertrauenspersonen: In immerhin jedem siebten Fall wusste die Mutter vom Missbrauch; halb so oft wussten Väter davon. Im institutionellen Kontext war dies deutlich seltener der Fall (Mutter: 6.0 \%; Vater: $4.0 \%$ im Vergleich zum nicht-institutionellen Kontext (Mutter: $22.6 \%$; Vater: $11.3 \%$ ). Außerdem lagen oftmals auch anderen Erziehungspersonen oder Großeltern bzw. anderen Verwandten Informationen vor.

Dass ein eingeleitetes Verfahren oder Ähnliches den Missbrauch beendete, dieser also aktiv durch Einschreiten dritter Personen unterbunden wurde, ist die Ausnahme: Nur 9.6\% aller Betroffenen geben an, dass sie jemandem vom Vorfall erzählt hätten und diese Person für das Ende des Missbrauchs gesorgt habe. Häufiger ging das Ende von der betroffenen Person selbst aus, sei es, weil sie weggezogen ist, weil das Ende des institutionellen Aufenthalts erreicht war, aus der Einrichtung geflohen wurde oder Ähnliches. Zudem werden täterbezogene Gründe benannt, beispielsweise dass die Täterin bzw. der Täter versetzt wurde (wobei unklar ist, ob dies Folge der Aufdeckung der Tat war oder nicht), sich einer anderen Person zugewandt hat, wegzog oder verstarb.

Mit 92.3\% hat die Mehrheit der Betroffen ihre Viktimisierungserfahrungen nach der Beendigung des Missbrauchs offenbart. Mehr als die Hälfte dieser Personen wartete dabei mehr als zehn Jahre, bevor es zur Offenbarung kam. Dabei gilt unabhängig davon, wem gegenüber sie sich offenbart haben, dass die Mehrheit der Betroffenen immer mehr als zehn Jahre gewartet hat, bis sie sich Dritten mitteilten. Besonders häufig wurden Freundinnen bzw. Freunde, Partnerinnen bzw. Partner oder Therapeutinnen bzw. Therapeuten ins Vertrauen gezogen. Immerhin 55.2 \% der Betroffenen wandten sich an Missbrauchsbeauftragte der katholischen Kirche; $36.2 \%$ haben über ihre Erlebnisse mit Journalistinnen bzw. Journalisten gesprochen.

Zwei Institutionen, an die sich die Betroffenen wandten, standen im Fokus differenzierterer Auswertungen: die katholische Kirche und die Polizei. Zwei Drittel der Betroffenen (68.0 \%) haben die katholische Kirche, ein Drittel (35.3 \%) die Polizei über die Geschehnisse informiert. Häufig genannte Gründe, warum sich die betroffenen Personen nicht an die Polizei gewandt haben, war der Eindruck, dass sie nichts beweisen könnten, dass sie nicht über das Geschehene sprechen könnten, dass sie niemanden bei der Polizei oder Staatsanwaltschaft kannten oder dass ihnen nicht geglaubt würde. 


\section{Das Urteil über die Unterstützung durch die katholische Kirche fällt ambivalent aus. Generell vermisst der Großteil der Be- troffenen eine ehrliche Auseinandersetzung der katholischen Kirche mit den Missbrauchstaten.}

Von denjenigen Personen, die sich an die katholische Kirche wandten, geben $84.3 \%$ an, dass ihnen geglaubt worden wäre. Zugleich berichtet fast die Hälfte der Betroffenen, dass danach nichts weiter passiert sei, dass sie abgewimmelt worden sei oder Ähnliches. Es werden also positive wie negative Erfahrungen geschildert. Für dieses ambivalente Bild können unterschiedliche Gründe verantwortlich sein: Einerseits ist durchaus vorstellbar, dass dieselbe Ansprechperson verschiedene Reaktionen zeigt, sich also beispielsweise zugewandt, interessiert und verständnisvoll verhält, gleichzeitig aber nichts weiter zur Untersuchung des Vorfalls unternimmt. Andererseits kann ein Kontakt mit mehreren Personen erfolgt sein, die sich unterschiedlich verhalten haben. Zudem ist zu beachten, dass sich die Betroffenen zu verschiedenen Zeitpunkten an die Kirche bzw. ihre Vertreterinnen und Vertreter gewandt haben. Dies wirkt sich den Daten zufolge auf die dabei gemachten Erfahrungen aus: Betroffene, die sich vor 2010 an die Kirche gewandt haben, berichten häufiger von negativen Erfahrungen als Betroffene, die sich nach 2010 dazu entschlossen haben. Umgekehrt ist der Anteil derer, die von positiven Erfahrungen berichten, bei denjenigen Betroffenen höher, die nach 2010 die katholische Kirche kontaktiert haben. Die öffentliche Debatte, die Einrichtung von Missbrauchsbeauftragten usw. gehen also mit einer den Betroffenen stärker zugewandten Haltung einher.

Etwas weniger als zwei Drittel der befragten Betroffenen, die die Kirche über den Missbrauch informiert haben, berichten, dass sie Entschädigungszahlungen erhalten hätten. Mit der Höhe der Zahlungen waren die meisten Betroffenen nicht zufrieden. Zugleich scheinen höhere Zahlungen nicht der wichtigste Wunsch der betroffenen Personen zu sein: 80.3 \% der Betroffenen äußern sich generell unzufrieden mit der Behandlung durch die Kirche. Die meisten Befragten vertreten die Ansicht, dass die Kirche nicht wirklich um Aufklärungsarbeit bemüht sei; es dominiert vielmehr der Eindruck, dass sie weiterhin daran interessiert sei, die Geschehnisse zu verdrängen und eine offene Auseinandersetzung zu vermeiden.

Dieser Eindruck wird unter anderem dadurch verstärkt, dass diejenigen Personen, die sich an die Kirche gewandt haben, selten erlebt haben, dass die Täterinnen und Täter sichtbar sanktioniert wurden. Eine Suspendierung wird nur von jeder fünften betroffenen Person mit Kirchenkontakt 
angegeben; ebenso hoch ist der Anteil derjenigen, die von einem eingeleiteten Disziplinarverfahren berichten. Eine Anzeige hat es nur in jedem 20. Fall gegeben (wohlgemerkt nur in Bezug auf jene Betroffenen, die sich an die Kirche gewandt haben). Eine Entschuldigung durch die Täterin bzw. den Täter erlebte ein nur unwesentlich höherer Prozentanteil der Betroffenen. Demgegenüber teilt jede dritte betroffene Person mit, dass die Täterin bzw. der Täter versetzt worden wäre. Es herrschte damit ein nachlässiger Umgang mit den Täterinnen und Tätern, was die Enttäuschung der Betroffenen verständlich machen dürfte. $\mathrm{Zu}$ beachten ist bei diesen Auswertungen allerdings, dass die Vorfälle bereits länger in der Vergangenheit liegen und dass auch aufgrund des Todes der Täterinnen und Täter bzw. der Verjährung der Tat formale Sanktionen seltener zur Anwendung gekommen sein könnten.

\section{Der Stellenwert des katholischen Glaubens im Leben der Be- troffenen ist ebenfalls ambivalent: Einerseits distanzieren sich die Betroffenen, andererseits lassen sich weiterhin Bindungen an katholische Glaubensvorstellungen feststellen.}

Zwei Drittel der Betroffenen sagen über sich, dass ihnen Religion und Glauben in der Zeit vor dem Missbrauch wichtig gewesen wären. Bis zum Zeitpunkt der Befragung hat sich dieser Anteil halbiert. Sehr deutlich zeigen sich Distanzierungsprozesse auch im Hinblick auf religiöse Aktivitäten wie das Beten oder den Kirchgang. Während die Betroffenen vor dem Missbrauch beispielsweise zu $48.1 \%$ mehrmals am Tag beteten, liegt der Anteil heute noch bei $10.6 \%$. Nur ein Drittel der vormaligen Mitglieder der katholischen Kirche war im Jahr der Befragung noch Mitglied in der katholischen Kirche, $69.9 \%$ sind aus der Kirche ausgetreten, wobei eine Häufung der Austritte nach 2010 festzustellen ist. Prinzipiell ist beim Vergleich der Zahlen zu beachten, dass Distanzierungsprozesse nicht allein mit dem Missbrauch in Zusammenhang stehen müssen. In den letzten 50 Jahren ist ebenso für die Allgemeinbevölkerung eine Säkularisierung festzustellen, die unter anderem mit einer Abkehr vom katholischen Glauben und einem allgemeinen Bedeutungsverlust kirchlich institutionalisierter Glaubenspraxis einhergeht (z. B. Breuer, 2012; Pollack \& Pickel, 2000). Zudem ist zu beachten, dass der hohe Anteil an Personen, die in Kindheit und Jugend religiöse Praktiken ausgeübt haben, zum Teil dadurch zustande kommt, dass fast jede bzw. jeder zweite Befragte im institutionellen 
Kontext missbraucht wurde, in denen diese Praktiken zur täglichen Pflicht gehörten.

Vor dem Hintergrund der schwerwiegenden Erlebnisse der Betroffenen ist eher überraschend, dass sich noch immer eine beachtliche Anzahl von ihnen als religiös einstuft und in der katholischen Kirche aktiv ist. Nicht nur für diese Gruppe gilt, dass die Distanzierung von Glaubensinhalten und -praktiken schwer zu fallen scheint. Die Bindung an den katholischen Glauben ist teils auch bei Personen erkennbar, die sich selbst als distanziert einstufen würden. Dies verdeutlichen die qualitativen Interviews: Fast alle Betroffenen rekurrieren in ihren biografischen Schilderungen auf religiöse Inhalte. Zahlen zum Kirchenaustritt, zur Kirchgangshäufigkeit usw. stellen insofern nur begrenzt gültige Indikatoren dazu dar, wie das Verhältnis zur Kirche beschaffen ist. Die Betroffenen wurden in ihrer Kindheit und Jugend stark religiös sozialisiert. Diese Erfahrungen prägen auch heute noch die Sichtweise auf verschiedene lebensgeschichtliche Phänomene. Der sich hier zeigende nachhaltige Einfluss religiöser Sozialisation sowie bestimmte Funktionen, die die Religion im biografischen Kontext erfüllt, führen letztlich dazu, dass die Betroffenen versuchen, das innerhalb der katholischen Kirche Erlebte mit den von dieser Kirche zur Verfügung gestellten geistigen Mitteln zu bewältigen. Dies ist in gewisser Weise ein Grundwiderspruch oder ein Grundkonflikt, der sich von den Betroffenen nur schwer auflösen lässt. Die hohen psychischen Belastungswerte, die für die betroffenen Personen insgesamt festzustellen sind, belegen dies.

\section{Eine positive elterliche Erziehung schützt vor sexuellem Miss- brauch; sie befähigt Betroffene des Missbrauchs zugleich häu- figer, sich hilfesuchend zu offenbaren.}

Die vorliegenden Befragungen richteten sich an Betroffene des sexuellen Missbrauchs im Kontext der katholischen Kirche. Parallel dazu erfolgte keine Befragung einer vergleichbaren Personengruppe, die keinen Missbrauch erlebt hat. Aus diesem Grund ermöglicht die Studie keine Aussagen über Risikofaktoren des Erlebens sexuellen Missbrauchs durch katholische Geistliche. Es kann damit nicht gesagt werden, unter welchen individuellen oder institutionellen Voraussetzungen es zum Missbrauch kommt und unter welchen nicht. Eine Auswertung gibt aber dennoch einen ersten Hinweis, wo mögliche Risikofaktoren zu suchen sind: in der Familie. Aus verschiedenen Studien ist bekannt, dass die familiäre Erzie- 
hung und hier insbesondere ein Mangel an Zuwendung und Kontrolle sowie die Anwendung von Gewalt mit problematischen Einstellungen und Verhaltensweisen einhergehen (z. B. Baier, Pfeiffer \& Thoben, 2013; Hellmann, 2014). Dies scheint auch für den sexuellen Missbrauch zu gelten.

Unter Rückgriff auf eine im Jahr 1992 durch das Kriminologische Forschungsinstitut Niedersachsen (KFN) durchgeführte Befragung ergibt sich, dass die Betroffenen weit häufiger über negative Erziehungserfahrungen berichten, als dies für die Allgemeinbevölkerung der Fall ist. Eine zugewandte, liebevolle Erziehung von Seiten der Eltern erlebten der vorliegenden Befragung zufolge $12.6 \%$ der Betroffenen sexuellen Missbrauchs, in der Allgemeinbevölkerung sind es 39.8\%. Vom Erleben schwerer elterlicher Gewalt in der Kindheit berichten $65.0 \%$ der Betroffenen, aber nur $16.2 \%$ der Personen der KFN-Befragung aus dem Jahr 1992. Bezogen auf das Vier-Faktoren-Modell von Finkelhor (1984), das unter anderem der Widerstandskraft der Kinder eine zentrale Rolle im Entstehungsprozess von Missbrauch zuweist, kann vermutet werden, dass die negativen familiären Erziehungserfahrungen die kindliche Widerstandskraft geschwächt haben. Die Kinder sind auf der Suche nach Zuwendung, die sie in der Familie nicht erhalten, und geraten dabei unter Umständen eher an Menschen, die ihre besonders vulnerable Lage ausnutzen.

Der Einfluss der elterlichen Erziehung zeigt sich zudem in zwei weiteren Auswertungen. So kann festgehalten werden, dass diejenigen Betroffenen, die von Seiten der Eltern liebevoller behandelt und dennoch durch einen katholischen Geistlichen sexuell missbraucht wurden, häufiger den Mut hatten, sich jemandem anzuvertrauen und damit aktiv den Missbrauch zu beenden. Zudem wirkt sich die Erfahrung elterlicher Gewalt auf die derzeitige psychische Befindlichkeit aus. Wer Gewalt in der Erziehung erlebt hat, weist signifikant schlechtere Befindlichkeitswerte auf als eine betroffene Person, für die dies nicht gilt. Der Einfluss der Erziehung reicht also über mehrere Jahrzehnte hinweg. Erklärbar sind die Effekte des elterlichen Erziehungsverhaltens unter anderem damit, dass sich positive wie negative Erziehungserfahrungen auf Persönlichkeitseigenschaften auswirken (z. B. Pfeiffer, Wetzels \& Enzmann, 1999). Hierzu gehören unter anderem Selbstkontrollfähigkeiten, das Ausmaß individueller Empathie und individueller Resilienz. 


\section{Anmerkungen zur Verallgemeinerbarkeit der Ergebnisse}

Einschränkend ist zu diesen zentralen Befunden darauf hinzuweisen, dass die Stichproben der Fragebogenuntersuchung und der Interviewstudie keine Repräsentativität beanspruchen können. Teilnehmerinnen und Teilnehmer der Fragebogenuntersuchung sind sämtlich Selbstmeldende, also Personen, die sich nach dem Presseaufruf des KFN an das Institut gewandt haben mit der Bitte, ihnen einen Fragebogen zuzusenden. Bei der Stichprobe der Interviewstudie handelt es sich ebenfalls um Selbstmeldende, wobei darunter auch Personen vertreten sind, die durch ein Bistum auf die Studie aufmerksam gemacht wurden und sich daraufhin erst an das KFN wandten. Insofern ist hier von einer heterogeneren Zusammensetzung des Samples der Interviewten auszugehen. Außerdem wurde im Rahmen der Interviewerhebung und -auswertung zusätzlich eine Auswahl durch das KFN getroffen, welche Fälle tatsächlich mit in die Untersuchung einbezogen werden sollen und welche nicht. Dabei wurde versucht, einerseits die Bandbreite an Missbrauchskonstellationen abzubilden. Andererseits wurden Fälle ausgewählt, mit deren Hilfe das Spannungsverhältnis zwischen Distanzierungsversuchen vom und Bindung an den katholischen Glauben beleuchtet werden sollte.

$\mathrm{Ob}$ die Stichprobe der Fragebogenuntersuchung tatsächlich in irgendeiner Weise verzerrt ist oder letztlich doch die Grundgesamtheit aller vom sexuellen Missbrauch betroffenen Personen abbildet, kann in Ermangelung einer Vergleichsstichprobe nicht beurteilt werden. Als wenig problematisch kann der überproportionale Anteil männlicher Betroffener eingestuft werden (75\% zu $25 \%$ ), insofern sich dies in anderen Studien (mit teilweise anderen methodischen Herangehensweisen) ebenso zeigt (z. B. John Jay College 2004; Lueger-Schuster, 2012; Zimmer, LappehsenLengler, Weber \& Götzinger, 2014). Auffällig sind zwei andere Befunde: Einerseits ist der Anteil an Betroffenen, die sich anderen Personen gegenüber geöffnet haben, mit - wie bereits erwähnt $-92.3 \%$ sehr hoch. Auch der Anteil derjenigen Betroffenen, die sich gegenüber der Kirche mitgeteilt haben, ist hoch (55.2\% Missbrauchsbeauftragte, $52.8 \%$ andere Personen der Kirche). Viele betroffene Personen haben sich zudem an Therapeutinnen bzw. Therapeuten, Beratungsstellen oder Journalistinnen bzw. Journalisten gewandt. Es entsteht damit der Eindruck, als handelte es sich bei den Befragten um jenen Teil der Betroffenen, der es - aus welchen Gründen auch immer - geschafft hat, über das Erlebte zu sprechen. Jene Missbrauchsbetroffenen, die diesen Schritt (noch) nicht vollziehen konnten, wären damit unterrepräsentiert. Andererseits zeigen die Angaben zum 
Zeitpunkt des Missbrauchs, dass sich in der Stichprobe keine Person befindet, die diesen nach 1993 erlebt hat. Es wäre naiv anzunehmen, dass es nach 1993 keine Fälle von Missbrauch gegeben hätte; die Betroffenen dürften aber weitestgehend noch nicht den Schritt gewagt haben, sich zu öffnen. Beide Befunde zusammengenommen lassen den Schluss zu, dass diejenigen Betroffenen, die aktuell am stärksten belastet sind, nicht Teil der Stichprobe sind. Insofern könnten die ohnehin hohen Anteile an belasteten Personen in der Stichprobe eine Unterschätzung darstellen. Dies ist zusätzlich deshalb zu vermuten, weil Personen, die sich aufgrund der Erlebnisse selbst getötet haben, ebenfalls nicht in der Stichprobe enthalten sind.

Während also im Hinblick auf die Befunde zu den Folgen des Missbrauchs die Ergebnisse der Fragebogenuntersuchung eher eine konservative Schätzung darstellen, dürften sie im Hinblick auf andere Befunde eher eine Überschätzung markieren. Dies ist unter anderem für die kritischen Einschätzungen zur Kirche zu vermuten. Es ist nicht von der Hand zu weisen, dass sich auf einen öffentlichen Aufruf zur Studienteilnahme, der nach dem Scheitern eines Forschungsprojekts mit dem Verband der Diözesen Deutschlands getätigt wurde, gerade jene Betroffenen melden und ihre Erfahrungen und Meinungen mitteilen möchten, die der katholischen Kirche gegenüber kritisch eingestellt sind.

In diesem Zusammenhang ist zusätzlich zu vermuten, dass sich Betroffene, die im Heim- und Internatskontext missbraucht wurden, häufiger an das KFN gewandt haben, weil der mediale Diskurs über den Missbrauch sehr stark auf diese Kontexte fokussiert war. Wie gezeigt, handelt es sich dabei um die eher schwerwiegenden Taten, die sich hier abgespielt haben. Was die Befunde zum Einsatz von Drohungen und Gewalt, zu den konkreten Tathandlungen oder zu den Täterinnen und Tätern anbelangt, könnten die erzielten Befunde damit ebenfalls kein völlig getreues Abbild der gesamten Missbrauchsrealität im Rahmen der katholischen Kirche darstellen. Es ist davon auszugehen, dass die dargestellten Ergebnisse damit eher die schwereren Übergriffe repräsentieren.

Auch wenn sich einige der genannten Einschränkungen nicht beheben ließen, wenn andere methodische Herangehensweisen gewählt würden, so wäre aus methodischer Sicht dennoch wünschenswert, dass es, um die Repräsentativität von derartigen Befragungsstudien zu erhöhen, eine Untersuchung nach dem Vorbild der Niederlande gäbe (Deetman et al., 2011). In einem Forschungsmodul wurde dabei eine Repräsentativbefragung von fast 35000 ab 40-jährigen Niederländerinnen und Niederländern durchgeführt. Darunter befanden sich 173 Befragte, die sexuellen Missbrauch 
durch katholische Geistliche erlebt haben. Diese 173 Befragten dürften die Betroffenengruppe besser repräsentieren als die ebenfalls im Rahmen des Projekts befragten 505 Betroffenen, die sich selbst gemeldet haben und mittels eines Fragebogens befragt wurden. Unter den 173 Befragten ist zum Beispiel der Anteil an betroffenen Personen höher, die ein nur einmaliges Erlebnis berichten, bei denen der Missbrauch damit von kürzerer Dauer war, sowie der Anteil derjenigen, die in der Gemeinde (das heißt nicht im institutionellen Kontext) missbraucht wurden.

\section{Ausblick}

Aus den Befunden lassen sich erste Folgerungen für die Prävention des Missbrauchs bzw. den Umgang mit den Betroffenen ableiten. Dies kann an dieser Stelle freilich nur sehr kursorisch erfolgen, da die Ableitung von Handlungsstrategien nicht nur voraussetzt, dass empirische, wissenschaftliche Befunde vorliegen, sondern ebenso, dass diese intensiv mit Praktikerinnen und Praktikern erläutert werden. In die Phase der vertieften Diskussion und Interpretation der Daten mit solchen Personen kann aber erst jetzt eingestiegen werden.

In Richtung der katholischen Kirche lassen die Befunde zumindest zwei zentrale Folgerungen zu: Erstens hat sich seit 2010 den Daten entsprechend die Art und Weise geändert, wie die Kirche den Betroffenen begegnet, die ihre Erlebnisse mitteilen. Dieser „Klimawandel“ - auch wenn er letztlich durch die öffentlich gewordenen Missbrauchsskandale ausgelöst wurde - sollte in jedem Fall weiter vollzogen werden. Eine wichtige Rolle scheinen dabei die Missbrauchsbeauftragten zu spielen. Es ist daher anzuraten, diese Anlaufstellen auch in Zukunft weiter zur Verfügung zu halten. Zweitens reicht es nicht aus, die Aufarbeitung des Missbrauchs auf die Arbeit dieser Personen zu beschränken. Es bedarf einer tiefgreifenden Auseinandersetzung mit der Problematik des sexuellen Missbrauchs. Dürfte es dabei prinzipiell leichter fallen, in Heimen und Internaten Kontrollen zur Vorbeugung von Missbrauch zu installieren, ist dies außerhalb dieser Kontexte innerhalb der katholischen Gemeinden deutlich schwieriger. Hier müssen sich die tätigen Personen mehr oder weniger selbst kontrollieren. Es gilt, in der Aus- und Weiterbildung verstärkt Sexualität und Missbrauch zu thematisieren, auch innerhalb der Kirche Ansprechpersonen für Geistliche zu schaffen und letztlich die eigenen Mitglieder zu motivieren, Fehlverhalten der Geistlichen (z. B. in anonymer Weise) zu melden. 
Ganz allgemein ist zu fordern, dass aufgrund der hohen psychischen Belastung der Betroffenen der Zugang zu therapeutischer Hilfe zu vereinfachen ist. Der Staat könnte dies im Rahmen des Opferentschädigungsgesetzes tun (siehe z. B. Hellmann \& Bartsch, 2014), was zugleich voraussetzt, dass die Betroffenen im Rahmen von Informationskampagnen über das Gesetz informiert werden (nur etwa die Hälfte der Befragten haben bislang davon gehört) und dass die Bewilligungsverfahren vereinfacht werden. Die Therapie selbst muss zugleich verstärkt die Besonderheiten des Missbrauchs durch katholische Geistliche berücksichtigen und dabei vor allem das Spannungsverhältnis von Distanzierung vom und Bindung an den katholischen Glauben thematisieren.

Abschließend ist noch einmal deutlich anzusprechen, dass es sich beim sexuellen Missbrauch durch katholische Geistliche nicht um ein abgeschlossenes Kapitel der katholischen Kirche handelt. Zwar liegen die berichteten Erlebnisse weitestgehend mehrere Jahrzehnte in der Vergangenheit. Daraus zu folgern, dass es entsprechende Übergriffe in den letzten Jahren nicht gegeben hat, wäre aber fahrlässig, und zwar deshalb, weil die Befunde klarmachen, wie schwer es den Betroffenen fällt, sich mitzuteilen und wie lange dies im Durchschnitt gedauert hat. Die katholische Kirche tut gut daran, sich weiterhin der Vergangenheit zu stellen, um für Gegenwart und Zukunft zu lernen und damit zu verhindern, dass es weitere Betroffene geben wird.

\section{Literatur}

Baier, D., Pfeiffer, C. \& Thoben, D. F. (2013). Elterliche Erziehung in Deutschland: Entwicklungstrends und Auswirkungen auf Einstellungen und Verhaltensweisen. Zeitschrift für Jugendkriminalrecht und Jugendhilfe, 24, 128-137.

Benkert, M. \& Doyle, T. P. (2009). Clericalism, religious duress and its psychological impact on victims of clergy sexual abuse. Pastoral Psychology, 58, 223-238.

Breuer, M. (2012). Religiöser Wandel als Säkularisierungsfolge. Differenzierungs- und Individualisierungsdiskurse im Katholizismus. Wiesbaden: VS.

Deetman, W., Draijer, N., Kalbfleisch, P., Merckelbach, H. Monteiro, M. \& de Vries, G. (2011). Sexual abuse of minors in the Roman Catholic church. Verfügbar unter: http://www.onderzoekrk.nl/eerste-onderzoek/eindrapport.html [04.02.2014]

Finkelhor, D. (1984). Child sexual abuse: New theory and research. New York, NY: Wiley.

Hellmann, D. F. (2014). Repräsentativbefragung zu Viktimisierungserfahrungen in Deutschland-Forschungsbericht Nr. 122. Hannover: KFN. 
Hellmann, D. F. \& Bartsch, T. (2014). Berücksichtigung der Belange von Betroffenen sexuellen Missbrauchs im Opferentschädigungsgesetz: Gleiches Recht für alle? Monatsschrift für Kriminologie und Strafrechtsreform, 97, 131-149.

John Jay College (2004). The nature and scope of sexual abuse of minors by Catholic priests and deacons in the United States, 1950-2002. Washington, DC: United States Conference of Catholic Bishops (USCCB).

Keupp, H., Straus, F., Mosser, P., Gmür, W. \& Hackenschmiedt, G. (2013). Sexueller Missbrauch, psychische und körperliche Gewalt im Internat der Benediktinerabtei Ettal. München: Institut für Praxisforschung und Projektberatung.

Lueger-Schuster, B. (2012). Psychotraumatologische Fragestellungen zu Gewalt und Missbrauch in der Katholischen Kirche. Wien: Universität Wien.

MacLean, K. (2003). The impact of institutionalization on child development. Development and Psychopathology, 15, 853-884.

Pfeiffer, C., Wetzels, P. \& Enzmann, D. (1999). Innerfamiliäre Gewalt gegen Kinder und Jugendliche und ihre Auswirkungen - Forschungsbericht Nr. 80. Hannover: KFN.

Plante, T. G. (1996). Catholic priests who sexually abuse minors: Why do we hear so much yet know so little? Pastoral Psychology, 44, 305-310.

Pollack, D. \& Pickel, G. (Hrsg.). (2000). Religiöser und kirchlicher Wandel in Ostdeutschland 1989-1999. Opladen: Leske + Budrich.

Stadler, L., Bieneck, S. \& Pfeiffer, C. (2012). Repräsentativbefragung sexueller Missbrauch-Forschungsbericht Nr. 118. Hannover: KFN.

Terry, K. J., Smith, M. L., Schuth, K., Kelly, J. R., Vollman, B. \& Massey, C. (2011). The causes and context of sexual abuse of minors by Catholic priests in the United States, 1950-2010. Washington, DC: United States Conference of Catholic Bishops (USCCB).

Whitelock, C. F., Lamb, M. E. \& Rentfrow, P. J. (2013). Overcoming trauma: Psychological and demographic characteristics of child sexual abuse survivors in adulthood. Clinical Psychological Science, 1, 351-362.

Zimmer, A., Lappehsen-Lengler, D., Weber, M. \& Götzinger, K. (2014). Sexueller Kindesmissbrauch in kirchlichen Institutionen - Zeugnisse, Hinweise, Prävention. Weinheim: Beltz. 